\title{
Systematic review and meta-analysis of surgical outcomes in Marfan patients undergoing aortic root surgery by composite- valve graft or valve sparing root replacement
}

\author{
Campbell D. Flynn ${ }^{1}$, David H. Tian ${ }^{2}$, Ashley Wilson-Smith ${ }^{2}$, Tirone David ${ }^{3}$, George Matalanis ${ }^{4}$, Martin $^{2}$ \\ Misfeld $^{5}$, Stefano Mastrobuoni ${ }^{6}$, Gebrine El Khoury ${ }^{6}$, Tristan D. Yan ${ }^{2,7,8}$ \\ ${ }^{1}$ Department of Cardiothoracic Surgery, Royal North Shore Hospital, St Leonards, Australia; ${ }^{2}$ Collaborative Research (CORE) Group, Macquarie \\ University, Sydney, Australia; ${ }^{3}$ Division of Cardiovascular Surgery, Peter Munk Cardiac Centre, Toronto General Hospital and University of \\ Toronto, Toronto, ON, Canada; ${ }^{4}$ Department of Cardiac Surgery, Austin Health, Melbourne, Australia; ${ }^{5}$ Cardiac Surgery, Heart Center Leipzig, \\ Leipzig, Germany; ${ }^{6}$ Department of Cardiovascular and Thoracic Surgery, Saint Luc's Hospital, Brussels, Belgium; ${ }^{7}$ University of Sydney, Royal \\ Prince Alfred Hospital, Sydney, Australia; ${ }^{8}$ University of Sydney, Sydney Adventist Hospital, Sydney, NSW, Australia \\ Correspondence to: Prof. Tristan D. Yan, MBBS, MD, PhD, FRACS. Director, Minimally Invasive Cardiothoracic Surgery, Sydney Adventist Hospital, \\ Suite 209, SAN Clinic Tulloch, 185 Fox Valley Road, Wahroonga, NSW, Australia. Email: tristanyan@annalscts.com.
}

Background: A major, life-limiting feature of Marfan syndrome (MFS) is the presence of aneurysmal disease. Cardiovascular intervention has dramatically improved the life expectancy of Marfan patients. Traditionally, the management of aortic root disease has been undertaken with composite-valve graft replacing the aortic valve and proximal aorta; more recently, valve sparing procedures have been developed to avoid the need for anticoagulation. This meta-analysis assesses the important surgical outcomes of the two surgical techniques.

Methods: A systematic review and meta-analysis of 23 studies reporting the outcomes of aortic root surgery in Marfan patients with data extracted for outcomes of early and late mortality, thromboembolic events, late bleeding complications and surgical reintervention rates.

Results: The outcomes of 2,976 Marfan patients undergoing aortic root surgery were analysed, 1,624 patients were treated with composite valve graft (CVG) and 1,352 patients were treated with valve sparing root replacement (VSRR). When compared against CVG, VSRR was associated with reduced risk of thromboembolism $(\mathrm{OR}=0.32 ; 95 \% \mathrm{CI}, 0.16-0.62, \mathrm{P}=0.0008)$, late hemorrhagic complications $(\mathrm{OR}=0.18$; 95\% CI, 0.07-0.45; P=0.0003) and endocarditis ( $\mathrm{OR}=0.27 ; 95 \% \mathrm{CI}, 0.10-0.68 ; \mathrm{P}=0.006)$. Importantly there was no significant difference in reintervention rates between VSRR and CVG (OR =0.89; 95\% CI, 0.35-2.24; $\mathrm{P}=0.80)$.

Conclusions: There is an increasing body of evidence that VSRR can be reliably performed in Marfan patients, resulting in a durable repair with no increased risk of re-operation compared to CVG, thus avoiding the need for systemic anticoagulation in selected patients.

Keywords: Marfan syndrome (MFS); valve sparing root; David procedure; Bentall; composite valve graft (CVG); total root replacement

Submitted Oct 27, 2017. Accepted for publication Nov 09, 2017.

doi: 10.21037/acs.2017.11.06

View this article at: http://dx.doi.org/10.21037/acs.2017.11.06 


\section{Introduction}

Marfan syndrome (MFS) is a genetic disorder of connective tissues with autosomal dominant inheritance due to mutation of the FBN1 gene, affecting approximately 1 in 5,000 patients. Diagnosis of the condition is made using the modified Ghent-2 criteria which provides a diagnosis based on clinical features, family history and genetic testing (1). Historically, patients with MFS had a median life expectancy of 49 years, limited largely due to complications of aortic pathology. Aneurysmal disease of the ascending aorta is particularly common with ascending aortic dilatation present in $53 \%$ of patients at 30 years. Life expectancy for Marfan patients is now similar to that of the general population, due to appropriate cardiovascular surgical intervention to minimize the risk of aortic complications (2).

Elective aortic root replacement is indicated in patients with MFS with an aortic root aneurysm $\geq 50 \mathrm{~mm}$ in diameter, or $\geq 45 \mathrm{~mm}$ when the patient has significant risk factors such as family history of acute aortic syndrome or rapid aneurysmal expansion of $>3 \mathrm{~mm} /$ year (3). Management of aortic root pathology necessitates resection of diseased aortic tissue, with the historical gold standard being total root replacement with a modified Bentall procedure as originally described in 1968 by Bentall and De Bono (4). This procedure replaces the aortic valve and proximal aorta with a composite valve graft (CVG), to which the coronary arteries are reimplanted. Due to the young age of Marfan patients, the use of a mechanical valve is often necessary, placing the patient at risk of thromboembolic and hemorrhagic complications. More recently, techniques to preserve the aortic valve for individuals with isolated aneurysmal disease and normal or mildly abnormal aortic valve leaflets have been developed $(5,6)$. These methods obviate the need for anticoagulation and avoid the risk of valverelated thromboembolism. However, a major concern of preserving aortic valve leaflets known to contain defective fibrillin-1 in MFS patients is the risk of early repair failure and need for re-operation (7). The concerns regarding the durability of valve sparing root replacement (VSRR) are being addressed with increased experience in the techniques and longer-term follow up data. Given the overall small number of MFS patients undergoing aortic root surgery, meta-analysis of the outcomes of observational studies provides the best evidence to support decision-making in this group of patients. To date, two meta-analyses have been undertaken by Benedetto et al. (8) in 2011 and $\mathrm{Hu}$ et al. (9) in 2014. This systematic review and meta-analysis aims to include an update of current published data and outcomes.

\section{Methods}

\section{Literature search strategy}

This meta-analysis was performed in accordance with PRISMA recommendations and guidance. The search strategy was employed to search electronic databases EMBASE, Ovid Medline, the entire Cochrane Central Register of Controlled Trails (CCRCT), Cochrane Database of Systematic reviews (CDSR), the Database of Abstracts of Reviews of Effects (DARE) and the ACP journal club from their inception to September 2017. The search strategy included search terms for ((aortic valve sparing or aortic valve preserving or David procedure) OR (Bentall or aortic root replacement or CVG) OR (aortic root surgery)) AND Marfan. The bibliography of previous systematic reviews was assessed to ensure no additional publications are missed.

\section{Selection criteria}

Eligibility for inclusion in this systematic review and metaanalysis included papers that assessed the outcomes of patients undergoing aortic root surgery in adult patients by either VSRR of any technique or total root replacement with CVG. In order to ensure sufficient institutional experience, papers were only included if more than 30 cases were reported. Studies were excluded if there was inadequate data regarding the outcomes of the repair technique or there was no separation of Marfan patient outcomes from the general patient outcomes. When centres reported outcomes of overlapping patient series then the most contemporary series was analysed. The analysis was limited to English language papers. Conference abstracts, case reports, editorials, expert opinion, reviews and expert opinion were excluded.

\section{Data extraction}

For the assessed papers, data was extracted from the reviewed text, tables and figures. Data was extracted independently by two of the authors (CDF and AWS) and any discrepancies were reviewed and discussed until consensus was reached. 
The recorded parameters were: number of cases in series, procedure undertaken, urgency of procedure, average age, average follow up, early death, late death, bleeding complications (late), systemic thromboembolic complications, post-operative endocarditis and reoperation.

\section{Statistical analysis}

Meta-analysis of incidence rates of post-operative complications, including endocarditis, thromboembolism, hemorrhagic complication and reoperation, was performed. The incidence rates were reported as number of events/ follow up year. Incidence rates were assessed drawing data from all papers. Incidence data was assessed using Comprehensive Meta-analysis v3.3. Comparative outcome data was determined only from observational studies reporting outcomes of both VSRR and CVG in MFS patients. Comparative outcomes were reported in events per follow-up year and were assessed using Review Manager v5.3 (Cochrane Collaboration, Copenhagen, Denmark). Data heterogeneity was assessed with the Cochrane Q statistic with $\mathrm{P}$ value $<0.05$ being significant and the $\mathrm{I}^{2}$ test statistic. Publication bias was assessed by generation of funnel plots and assessment using Egger's test. The number of patient years was calculated by multiplying the number of patients included in the study by the average follow up duration of that study. Annual event rates are determined from data on all included studies, with no comparison made between CVG and VSRR on overall event rate data due to the risk of heterogeneity of data. Comparative outcomes are determined from subgroup random-effects analysis of the included studies reporting outcomes of both CVG and VSRR to determine the effect of surgical technique on outcomes. Sensitivity analysis was conducted for all assessed variables using 'remove-one' analysis. Individual patient survival data was reconstructed using an iterative algorithm that was applied to digitized source Kaplan-Meier curves and subsequently aggregated and graphed (10).

\section{Results}

The search strategy revealed 900 citations for review after duplicates were removed, 23 of which met the predetermined inclusion criteria (11-33) (Figure S1). The included publications had a total patient population of 2,976; 1,624 patients were treated with CVG and 1,352 patients were treated with VSRR. For all patients, the total number of patient-years follow up for CVG is
8,794 and the total number of patient-years follow up for VSRR is 5,741. The average follow-up for period for CVG in 13 studies reporting follow up duration was 7.14 years and the average follow up period for VSRR in 20 studies reporting follow up duration was 4.5 years. There were 2 studies reporting the outcomes of CVG only $(11,19)$ and 8 studies reporting the outcomes of VSRR only $(15,17,20,22,23,26,28,31)$. Thirteen studies reported outcomes for both CVG and VSRR $(12-14,16,18,21,24,25,27,29,30,32,33)$. The overall study characteristics are detailed in Table 1.

Assessing overall data, the incidence of late bleeding complications for CVG was $1.3 \%$ (95\% CI, $0.4-2.1 \%$ ) events/follow up year and the incidence of thromboembolic phenomena was $0.7 \%$ (95\% CI, $0.5-0.9 \%$ ) events/ follow up year. The incidence of late bleeding complications and thromboembolic phenomena for VSRR was $0.1 \%$ (95\% CI, $0.0-0.3 \%$ ) and $0.4 \%$ (95\% CI, $0.3-0.5 \%)$ event/follow-up year respectively. The incidence of re-operation for CVG and VSRR was $1.3 \%$ (95\% CI, $-1.0-3.7 \%)$ and $0.6 \%$ (95\% CI, $0.3-0.9 \%$ ) events/follow up year respectively. Full event rates for surgical outcomes are detailed in Table 2 .

Meta-analysis of comparative studies demonstrated a significant reduction in late mortality for patients undergoing VSRR (OR =0.26; 95\% CI, 0.15-0.46; $\mathrm{P}<0.0001)$ (Figure 1). As expected, there was a reduced risk of thromboembolic events (Figure 2) for VSRR (OR $=0.32 ; 95 \% \mathrm{CI}, 0.16-0.62 ; \mathrm{P}=0.0008)$ and late hemorrhagic complications (Figure 3) $(\mathrm{OR}=0.18$; 95\% CI, 0.07-0.45; $\mathrm{P}=0.0003)$. The odds of post-operative endocarditis were less after VSRR than after CVG (Figure 4) $(\mathrm{OR}=0.27$; 95\% CI, 0.10-0.68; $\mathrm{P}=0.006$ ). Importantly, the risk of reoperation was not increased (Figure 5) for VSRR when compared to $\mathrm{CVG}(\mathrm{OR}=0.89 ; 95 \% \mathrm{CI}, 0.35-2.24 ; \mathrm{P}=0.80$ ), however this was a high level of heterogeneity between studies $\left(\mathrm{I}^{2}=60 \%\right)$, sensitivity analysis did not reveal any change in combined effect. Follow-up duration of VSRR and CVG did not explain any difference between VSRR and CVG on meta-regression analysis $\left(\mathrm{R}^{2}=0.00\right)$.

The congregate Kaplan-Meyer curve for overall survival for CVG and VSRR is shown in Figure 6. Overall survival at 1-, 2-, 3-, 5-, and 10-year was $97.6 \%, 95.9 \%, 95.1 \%$, $92.4 \%$, and $84.5 \%$, respectively. Congregate Kaplan-Meyer curves for overall re-intervention rate for CVG and VSRR (Figure 7) was 97.8\%, 97.0\%, 96.2\%, 95.6\%, and 90.5\%, respectively at 1-, 2-, 3-, 5-, and 10-year.

There was no evidence of publication bias in any of the reported outcomes using Egger's test or through visual 


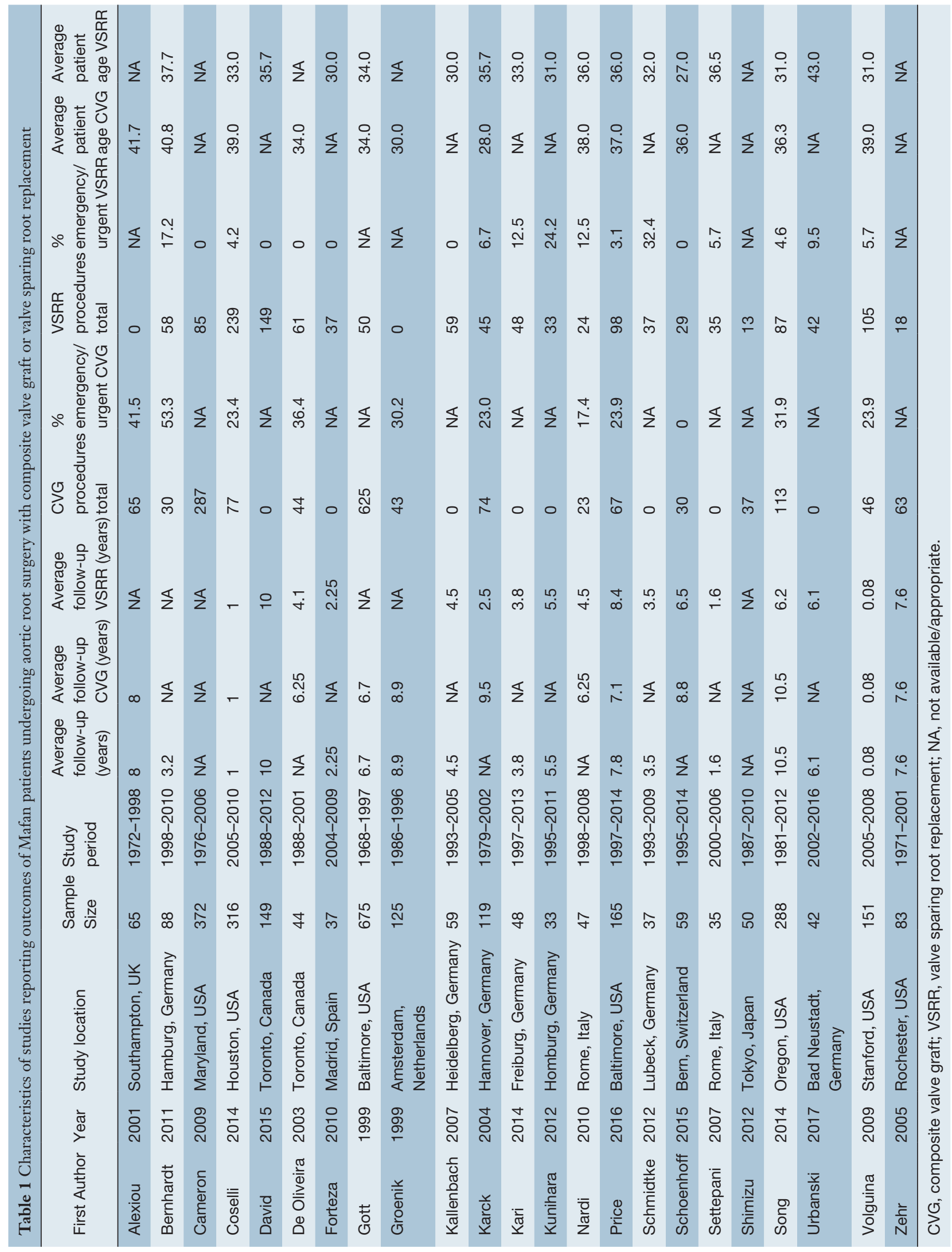




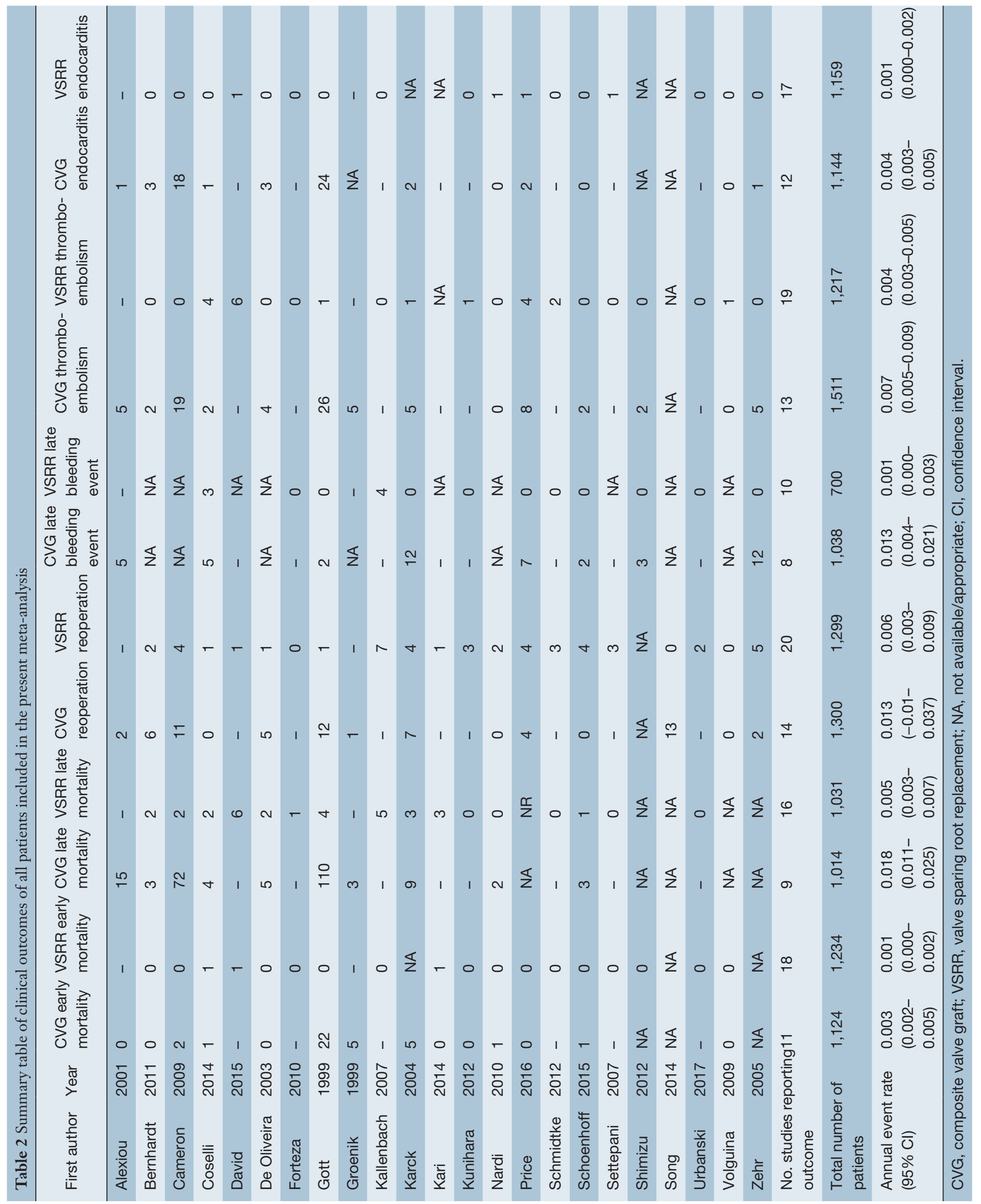




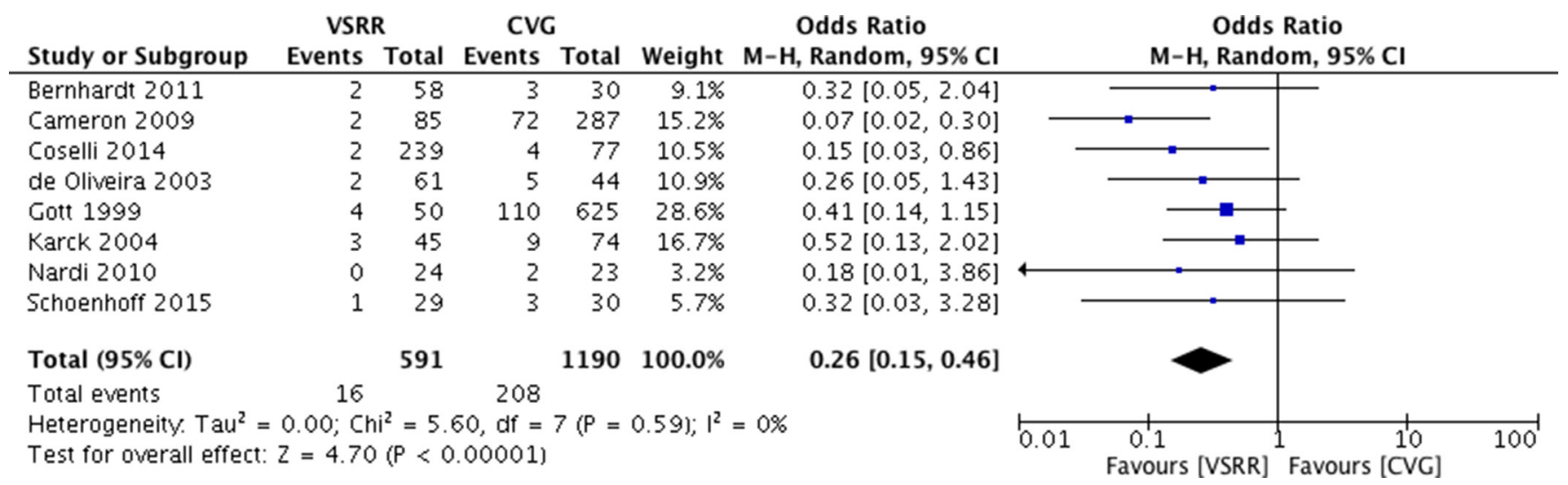

Figure 1 Comparison of late mortality between CVG and VSRR. CVG, composite valve graft; VSRR, valve sparing root replacement.

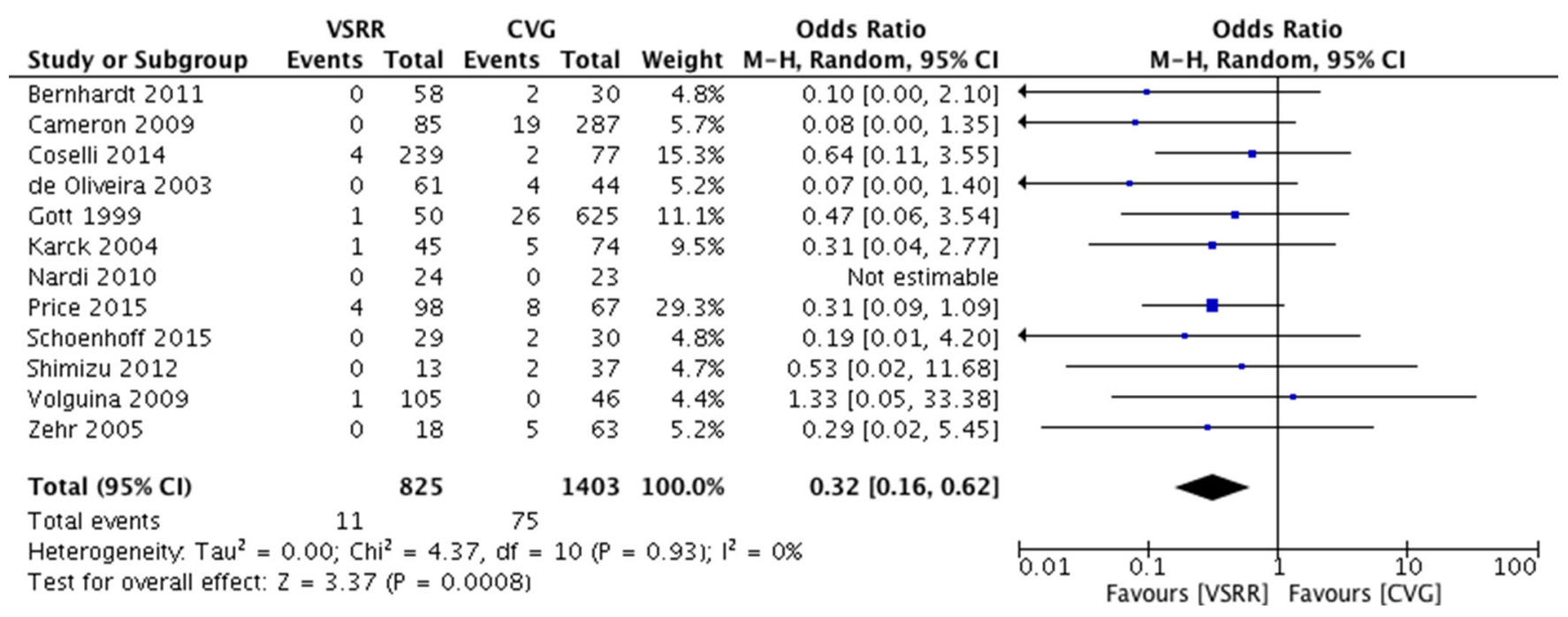

Figure 2 Comparison of thromboembolic events between CVG and VSRR. CVG, composite valve graft; VSRR, valve sparing root replacement.

\section{VSRR CVG Odds Ratio} Study or Subgroup Events Total Events Total Weight $\mathrm{M}-\mathrm{H}, \mathrm{Random}, \mathbf{9 5 \%} \mathrm{Cl}$

\begin{tabular}{lrrrrrr} 
Coselli 2014 & 3 & 239 & 5 & 77 & $40.8 \%$ & \\
Cott 1999 & 0 & 50 & 2 & 625 & $9.3 \%$ & 2.47 \\
Karck 2004 & 0 & 45 & 12 & 74 & $10.6 \%$ \\
Price 2015 & 0 & 98 & 7 & 67 & $10.4 \%$ \\
Schoenhoff 2015 & 0 & 29 & 2 & 30 & $9.1 \%$ \\
Shimizu 2012 & 0 & 13 & 3 & 37 & $9.4 \%$ \\
Zehr 2005 & 0 & 18 & 12 & 63 & $10.4 \%$ \\
Total (95\% CI) & & 492 & & 973 & $100.0 \%$ \\
Total events & 3 & \multicolumn{5}{c}{43} \\
Heterogeneity. Tau & $=0.00 ; \mathrm{Chi}^{2}=5.14, \mathrm{dr}=6(\mathrm{P}=0.53) ; \mathrm{I}^{2}=0 \%$ \\
Test for overall effect: $\mathrm{Z}=3.63(\mathrm{P}=0.0003)$ &
\end{tabular}

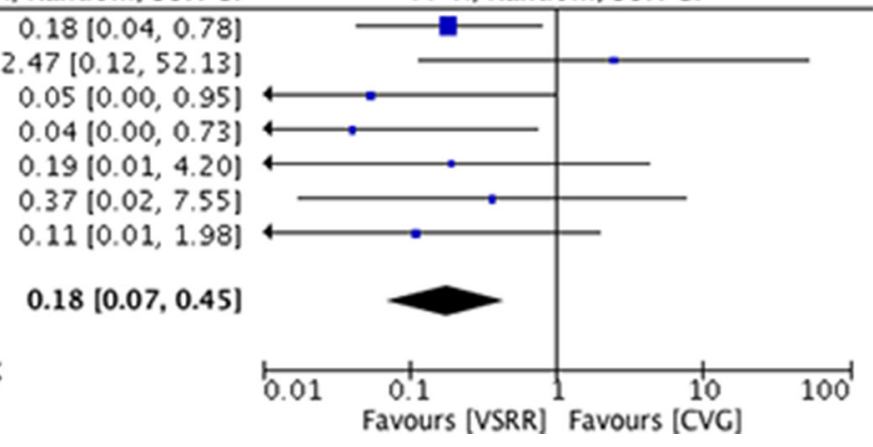

Figure 3 Comparison of late haemorrhagic events between CVG and VSRR. CVG, composite valve graft; VSRR, valve sparing root replacement. 


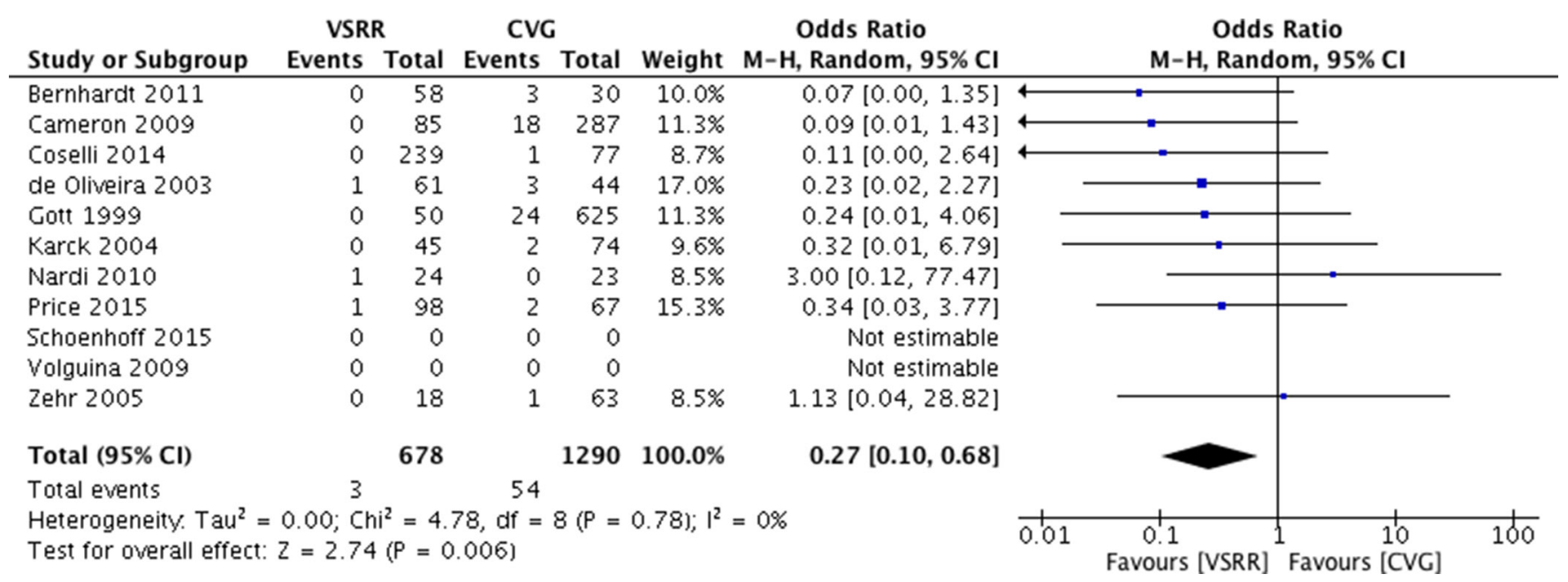

Figure 4 Comparison of endocarditis events between CVG and VSRR. CVG, composite valve graft; VSRR, valve sparing root replacement.

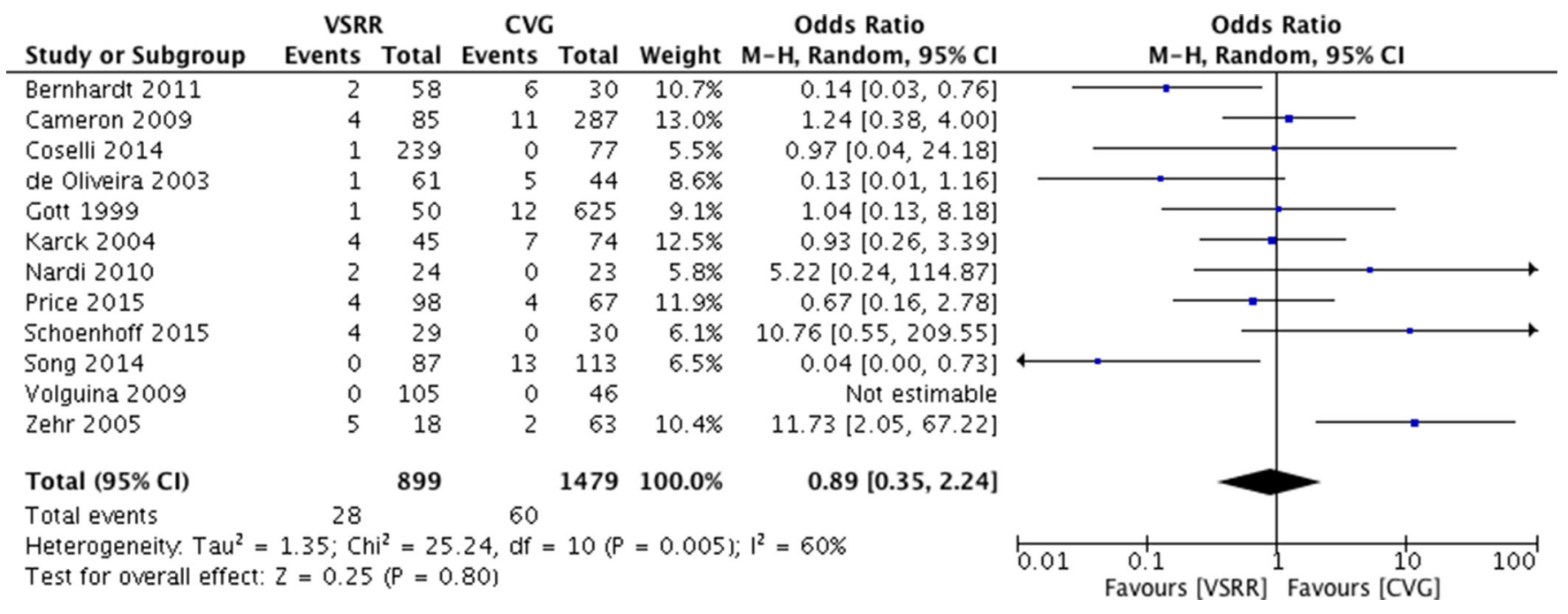

Figure 5 Comparison of surgical re-intervention between CVG and VSRR. CVG, composite valve graft; VSRR, valve sparing root replacement.

inspection of funnel plots (Figures S2-S7).

\section{Discussion}

Vascular intervention in patients with MFS is responsible for the recent and substantial improvement in the lifeexpectancy of this young population. For a condition affecting a small proportion of the population, the prospect of a high quality randomized control trial to provide evidence is unlikely. However, by pooling data from observational studies for meta-analysis, we are able to improve the evidence-base upon which we make decisions for intervention strategy. The present study has drawn data from a pool of 2,976 patients from experienced surgical centers to determine incidence rates for important postoperative complications and data from 2,457 patients in studies that provide a comparison of outcomes of composite valve-graft and valve-sparing root replacement within the same institution.

This meta-analysis has demonstrated the potential longterm benefit of Marfan patients undergoing valve-sparing root replacement over composite valve grafts. Significant 


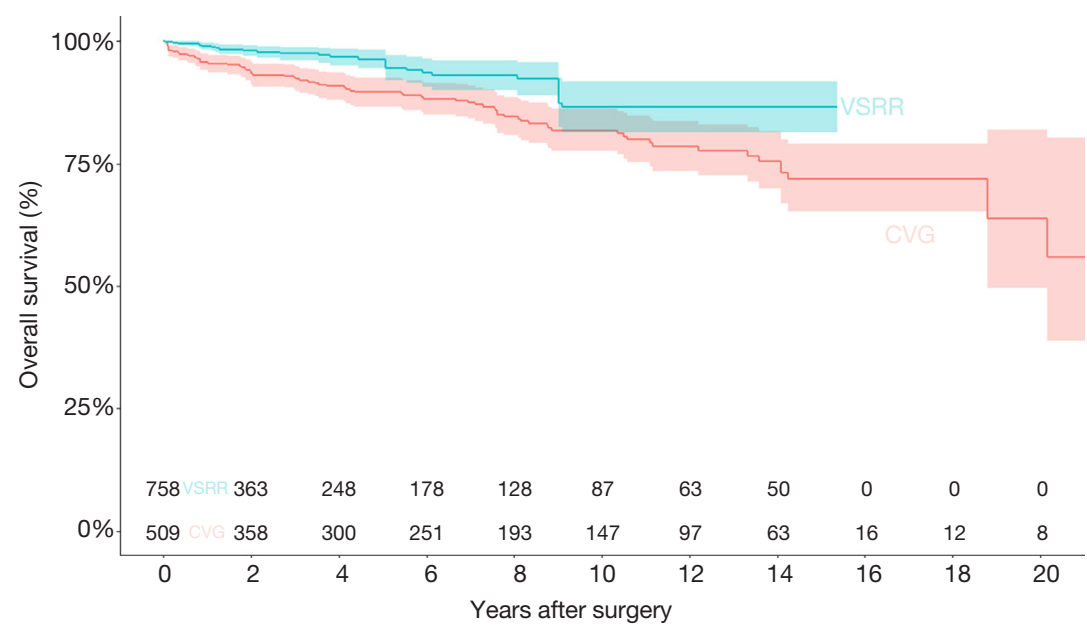

Figure 6 Congregate Kaplan-Meier curve for overall survival between CVG and VSRR. Shaded region represents $95 \%$ confidence interval. CVG, composite valve graft; VSRR, valve sparing root replacement.

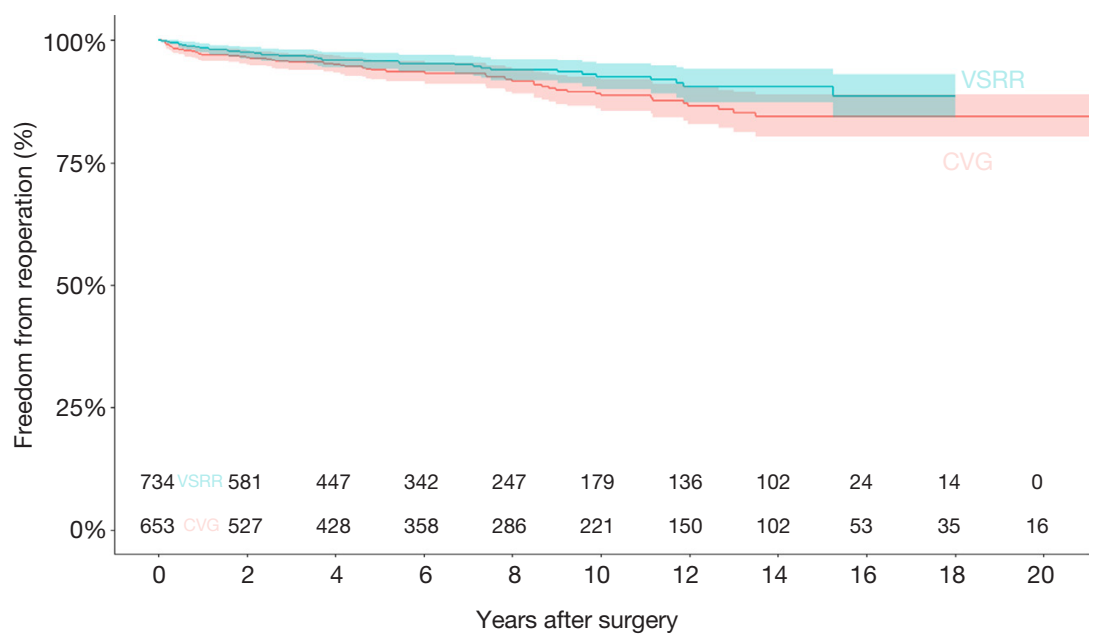

Figure 7 Congregate Kaplan-Meier curve for late re-operation for VSRR and CVG. Shaded region represents $95 \%$ confidence interval. VSRR, valve sparing root replacement; CVG, composite valve graft.

benefit of VSRR for thromboembolic complications have previously been demonstrated in meta-analysis $(8,9)$, the latter study by $\mathrm{Hu}$ et al. in 2014 (9) also favoured VSRR for endocarditis rates. Interestingly, Benedetto et al. (8) reported a four-fold increased risk of intervention on aortic root with VSRR compared to CVG, a finding not supported by the present meta-analysis or by $\mathrm{Hu}$ et al. This disparity may be explained by the greater sample sizes available in the more recent studies.

The traditional gold standard of composite valve graft for the management of aortic root disease provides an excellent improvement in the survival of Marfan patients. However, due to their young age at the time of surgery, they usually require the use of a mechanical prosthesis and systemic anticoagulation which puts the individual at risk of hemorrhagic and thromboembolic complications as well as an increased risk of endocarditis. Furthermore, female patients may be of childbearing age, making the provision of systemic anticoagulation challenging.

Limitations in current data stem from the observational and largely retrospective nature of the data analyzed which increases the risk of bias and potentially decreases the 
accuracy of collected information. Volguina and Coselli reported early outcomes of a multicenter, prospectively designed study of aortic surgery in Marfan patients from centers in Northa America, South America and Europe $(14,32)$. This study along with the GenTAC registry (34), a prospectively designed multicenter registry for genetically mediated thoracic aneurysmal disease will certainly add to the quality of evidence to guide decision making when longterm data becomes available.

An important concession for the decision-making process is that valve-sparing root replacement is by no means appropriate for every Marfan patient undergoing aortic surgery as both surgeon and patient factors may necessitate a total root replacement with composite valve-graft. Not all patients are appropriate for valve-sparing root replacement as the presence of leaflet fenestration or tissue redundancy may make a satisfactory and durable repair impossible. This inherent selection bias will be impossible to eliminate from any future study. Furthermore, because valve sparing surgery is a technically demanding procedure, centers with low volumes are likely to have worse outcomes (35). Therefore the procedure should be carried out in centers with sufficient expertise.

\section{Expert opinion 1 (Tirone E. David)}

This is a timely meta-analysis on the surgical outcomes of patients with MFS undergoing aortic root surgery by composite-valve graft (CVG) or VSRR. Flynn and colleagues reviewed 900 references and identified 23 studies that they used to compare the outcomes of 1,624 patients with MFS treated with CVG with 1,352 patients who had VSRR. The average follow-up was longer for CVG (7.1 years) than for VSRR (4.5 years) patients. As expected, rates of bleeding and thromboembolism were higher in CVG than in VSRR patients. Unexpectedly, the rate of reoperation was also higher in CVG than in VSRR, but as pointed out by the authors there was a high level of heterogeneity between studies. I would have expected a higher rate of reoperation after VSRR.

I believe that patients with aortic root aneurysm associated with genetic syndromes should have VSRR whenever possible. Actually, it is feasible in most patients because surgery is recommended early in the course of aortic sinuses dilatation when the cusps are still normal or have minor structural abnormalities (36). I have, and continue to be very conservative in all patients with aortic root aneurysm and perform VSRR only when the cusps are normal or have only minor abnormalities such as elongation of the free margin or small stress fenestrations along the commissural areas (37). This conservative approach may explain my exceptionally good long-term results as they relate to reoperation and the development of aortic insufficiency $(36,37)$.

CVG was introduced two decades earlier than VSRR and it is a more reproducible operation than VSRR. The main drawback of CVG is that mechanical valves should be used in patients with MFS because they are often in their second or third decades of life by the time they need aortic root surgery and mechanical valves require lifelong anticoagulation with warfarin. Tissue valves in these patients have limited durability.

\section{Expert opinion 2: “Don't throw the baby out with the bathwater" (George Matalanis)}

The general philosophy of VSRR is the provision of a hemodynamically efficient aortic valve with durability exceeding that of a bioprosthesis, no requirement for anticoagulation and low risk of long term complications such as infection. Because most Marfan's patients present for surgery at a young age, the avoidance of anticoagulation is not only desirable for considerations such as life style and pregnancy, but also from the inevitable need for subsequent cardiac and non-cardiac surgery. The greater exercise demands in this patient group also benefit from the absence of significant gradients or regurgitation from a well performed VSRR. Nonetheless, in the context of the well described Marfan's fibrillin deficiency and frequent occurrence of fenestrations there is a genuine concern regarding durability.

In this meta-analysis, Flynn and colleagues performed a systematic review of the available literature to elucidate both the acute and chronic performance of VSSR $v s$. CVG. Importantly, the operative mortality was very low and equivalent in both groups, as expected from this young and healthy cohort. Thus, the extra manipulations incurred in performing a VSRR were not resulting in extra operative risk. The rest of the findings supported better outcomes with VSRR including, lower long-term bleeding, thromboembolic and infective complications than CVG. All this was achieved without a significant trade off of increased late re-operation rate.

In the midst of such salutary findings in favour of valve preservation in the Marfan's root, a word of caution is in order. Firstly, it is sobering to appreciate that such 
great long-term outcomes of VSRR would have easily been lost had the early morbidity and mortality not been so excellent in the reported series. Such results are achieved in experienced centers due to a combination of careful case selection and surgical familiarity with the techniques. Clearly, there are Marfan's aortic valves with numerous fenestrations and extreme fragility, that would be inappropriate to preserve. Equally obvious, as pointed out by the authors, there was a degree of variability in the freedom for re-intervention between the series. Therefore, we will have to wait for larger numbers followed for longer periods to be absolutely sure of the longevity of VSRR in Marfan's syndrome.

The authors have laid down a firm evidence basis for us to recommend VSRR in centres with good experience in the repair techniques, with the same confidence as mitral valve repair in Marfan's syndrome.

\section{Expert opinion 3: selection and expertise is the key to success (Martin Misfeld)}

Complications of aneurysmal disease limits the life expectancy of patients with MFS. Replacement of the aortic root either by CVG replacement or by VSRR have led to excellent surgical results.

This systematic review and meta-analysis of patients with MFS was performed by Flynn $e t a l$. with a total number of 23 studies, incorporating 1,624 CVG and 1,352 VSRR patients. The was no statistically significant difference in reintervention rates. There was a significantly reduced risk of thrombembolism, late hemorrhagic complications and endocarditis in patients with VSRR.

A classic phenotypic expression of MFS affects mutations of the gene encoding fibrillin-1. Recent genetic studies demonstrate a high degree of overlap between MFS other connective tissue diseases (e.g., Loeys-Dietz syndrome, Ehlers-Danlos syndrome, and others). There are several variations of clinical phenotype in patients with MFS, even intra-familial. Therefore, the diagnosis of MFS may not represent a uniform disease and the clinical picture and indication for surgery may also vary.

In this manuscript, VSRR has been shown to have advantages compared to CVG, although there is clearly a selection bias. VSRR itself contains the aortic valve remodelling and the reimplantation technique. Stabilization of the aortic root annulus is a key factor in MFS patients. Therefore, either the reimplantation technique or the remodelling technique with additional annular support should be performed. Surgical expertise is essential when performing this procedure, particularly when repair to aortic valve cusps is required. Regular follow-up in qualified centers for survillance of the residual aorta and dealing with concomitant diseases (e.g., skeletal and eyes) as well as genetic and family counseling is required.

As the authors rightly state, patients with MFS should be treated in centers with experience mangaging this complicated group of patients. This allows for appropriate decision making for surgery, enables additional support for the management of concomitant diseases and an adequate surgical expertise to perform either CVG or VSRR to obtain the excellent results presented in this paper.

\section{Expert opinion 4 (Stefano Mastrobuoni and Gebrine El Khoury)}

Dr. Flynn and colleagues have performed a systematic review and meta-analysis of studies on the surgical outcomes of VSRR versus traditional operation (Bentall operation) with valve prosthesis in patients with Marfan syndrome. The current meta-analysis is particularly important because since a previous analysis by Benedetto (8) in 2011 that questioned the efficacy of VSRR in this selective cohort of patients, several important papers have been published which have been included by Flynn and colleagues. Indeed, since then, Dr. David (15) published his experience with Marfan patients as well as the groups of Dr. Cameron (25) in Baltimore (US), Dr. Carrel (27) in Bern (Switzerland), Dr. Sievers (26) in Lubeck (Germany) and others. The current meta-analysis shows clearly that VSRR is associated with particularly low rates of valve-related complications such as bleeding, thromboembolism, endocarditis and an improved survival compared to the conventional Bentall operation. The relative risk reduction is stunningly in the order of $70 \%$ for these complications. It seems unlikely that future studies may overturn these results. Further, the current analysis did not reveal an increased risk of reoperation after VSRR that is usually considered a major limitation of this operation.

Usually Marfan patients arrive at surgery in their 3rd or 4th decade of life. In this cohort of young patients requiring root and ascending aorta replacement the choice is between a composite graft with either a mechanical or a tissue prosthesis, VSRR or a Ross operation. A composite graft with a tissue prosthesis at this age expose the patient to a high risk of reoperation in the mid-term and a decreased survival, and therefore offers no advantages compared to 
VSRR. Similarly, considering the underlying connective tissue disorder and the complexity of the procedure, the Ross operation is not an attractive option either. Thus, the choice really is between VSRR and mechanical prosthesis. We believe that the reduction of valve-related complications associated with mechanical prosthesis and life-long oral anticoagulation is of particular interest in order to guarantee the quality of life of these young, active patients even if they may have a certain risk of reoperation over time. Nonetheless, in experienced centers, reoperations after VSRR are routinely performed with low morbidity and mortality. Moreover, we have observed that Marfan patients present for surgery early in the disease course and large fenestrations or other cusp diseases that preclude an effective repair are rarely seen. Therefore, in elective Marfan patients VSRR can be safely performed with excellent results.

\section{Acknowledgements}

None.

\section{Footnote}

Conflicts of Interest: The authors have no conflicts of interest to declare.

\section{References}

1. Loeys BL, Dietz HC, Braverman AC, et al. The revised Ghent nosology for the Marfan syndrome. J Med Genet 2010;47:476-85.

2. Silverman DI, Burton KJ, Gray J, et al. Life expectancy in the Marfan syndrome. Am J Cardiol 1995;75:157-60.

3. Erbel R, Aboyans V, Boileau C, et al. 2014 ESC Guidelines on the diagnosis and treatment of aortic diseases: Document covering acute and chronic aortic diseases of the thoracic and abdominal aorta of the adult. The Task Force for the Diagnosis and Treatment of Aortic Diseases of the European Society of Cardiology (ESC). Eur Heart J 2014;35:2873-926.

4. Bentall H, De Bono A. A technique for complete replacement of the ascending aorta. Thorax 1968;23:338-9.

5. David TE, Feindel CM. An aortic valve-sparing operation for patients with aortic incompetence and aneurysm of the ascending aorta. J Thorac Cardiovasc Surg 1992;103:61721; discussion 22.

6. Sarsam MA, Yacoub M. Remodeling of the aortic valve anulus. J Thorac Cardiovasc Surg 1993;105:435-8.
7. Fleischer KJ, Nousari HC, Anhalt GJ, et al. Immunohistochemical abnormalities of fibrillin in cardiovascular tissues in Marfan's syndrome. Ann Thorac Surg 1997;63:1012-7.

8. Benedetto U, Melina G, Takkenberg JJ, et al. Surgical management of aortic root disease in Marfan syndrome: a systematic review and meta-analysis. Heart 2011;97:955-8.

9. Hu R, Wang Z, Hu X, et al. Surgical reconstruction of aortic root in Marfan syndrome patients: a systematic review. J Heart Valve Dis 2014;23:473-83.

10. Guyot P, Ades AE, Ouwens MJ, et al. Enhanced secondary analysis of survival data: reconstructing the data from published Kaplan-Meier survival curves. BMC Med Res Methodol 2012;12:9.

11. Alexiou C, Langley SM, Charlesworth P, et al. Aortic root replacement in patients with Marfan's syndrome: the Southampton experience. Ann Thorac Surg 2001;72:15027; discussion 8 .

12. Bernhardt AM, Treede H, Rybczynski M, et al. Comparison of aortic root replacement in patients with Marfan syndrome. Eur J Cardiothorac Surg. 2011;40:1052-7.

13. Cameron DE, Alejo DE, Patel ND, et al. Aortic root replacement in 372 Marfan patients: evolution of operative repair over 30 years. Ann Thorac Surg 2009;87:1344-9; discussion 9-50.

14. Coselli JS, Volguina IV, LeMaire SA, et al. Early and 1-year outcomes of aortic root surgery in patients with Marfan syndrome: a prospective, multicenter, comparative study. J Thorac Cardiovasc Surg 2014;147:1758-66, 67.e1-4.

15. David TE, David CM, Manlhiot C, et al. Outcomes of Aortic Valve-Sparing Operations in Marfan Syndrome. J Am Coll Cardiol 2015;66:1445-53.

16. de Oliveira NC, David TE, Ivanov J, et al. Results of surgery for aortic root aneurysm in patients with Marfan syndrome. J Thorac Cardiovasc Surg 2003;125:789-96.

17. Forteza A, De Diego J, Centeno J, et al. Aortic valve-sparing in 37 patients with Marfan syndrome: midterm results with David operation. Ann Thorac Surg 2010;89:93-6.

18. Gott VL, Greene PS, Alejo DE, et al. Replacement of the aortic root in patients with Marfan's syndrome. N Engl J Med 1999;340:1307-13.

19. Groenink M, Lohuis TA, Tijssen JG, et al. Survival and complication free survival in Marfan's syndrome: implications of current guidelines. Heart 1999;82:499-504.

20. Kallenbach K, Baraki H, Khaladj N, et al. Aortic valvesparing operation in Marfan syndrome: what do we know after a decade? Ann Thorac Surg 2007;83:S764-8; discussion S85-90. 
21. Karck M, Kallenbach K, Hagl C, et al. Aortic root surgery in Marfan syndrome: Comparison of aortic valve-sparing reimplantation versus composite grafting. J Thorac Cardiovasc Surg 2004;127:391-8.

22. Kari FA, Beyersdorf F, Rylski B, et al. David I reimplantation procedure for aortic root replacement in Marfan patients: medium-term outcome. Interact Cardiovasc Thorac Surg 2014;19:743-8.

23. Kunihara T, Aicher D, Rodionycheva S, et al. Outcomes after valve-preserving root surgery for patients with Marfan syndrome. J Heart Valve Dis 2012;21:615-22.

24. Nardi P, Pellegrino A, Versaci F, et al. Aortic root surgery in Marfan syndrome: Bentall procedure with the composite mechanical valved conduit versus aortic valve reimplantation with Valsalva graft. J Cardiovasc Med (Hagerstown) 2010;11:648-54.

25. Price J, Magruder JT, Young A, et al. Long-term outcomes of aortic root operations for Marfan syndrome: A comparison of Bentall versus aortic valve-sparing procedures Read at the 95th Annual Meeting of the American Association for Thoracic Surgery, Seattle, Washington, April 25-29, 2015. J Thorac Cardiovasc Surg 2016;151:330-6.

26. Schmidtke C, Karluss A, Sier H, et al. Mid-term results of different aortic valve-sparing procedures in Marfan syndrome. J Heart Valve Dis 2012;21:195-201; discussion 2.

27. Schoenhoff FS, Langhammer B, Wustmann K, et al. Decision-making in aortic root surgery in Marfan syndrome: bleeding, thromboembolism and risk of reintervention after valve-sparing or mechanical aortic root replacement. Eur J Cardiothorac Surg 2015;48:931-5; discussion 5-6.

28. Settepani F, Szeto WY, Pacini D, et al. Reimplantation valvesparing aortic root replacement in Marfan syndrome using the Valsalva conduit: an intercontinental multicenter study. Ann Thorac Surg 2007;83:S769-73; discussion S85-90.

Cite this article as: Flynn CD, Tian DH, Wilson-Smith A, David T, Matalanis G, Misfeld M, Mastrobuoni S, El Khoury G, Yan TD. Systematic review and meta-analysis of surgical outcomes in Marfan patients undergoing aortic root surgery by composite-valve graft or valve sparing root replacement. Ann Cardiothorac Surg 2017;6(6):570-581. doi: 10.21037/ acs.2017.11.06
29. Shimizu H, Kasahara H, Nemoto A, et al. Can early aortic root surgery prevent further aortic dissection in Marfan syndrome? Interact Cardiovasc Thorac Surg 2012;14:171-5.

30. Song HK, Preiss LR, Maslen CL, et al. Valve-sparing aortic root replacement in patients with Marfan syndrome enrolled in the National Registry of Genetically Triggered Thoracic Aortic Aneurysms and Cardiovascular Conditions. J Heart Valve Dis 2014;23:292-8.

31. Urbanski PP, Jankulowski A, Morka A, et al. Patienttailored aortic root repair in adult marfanoid patients: Surgical considerations and outcomes. J Thorac Cardiovasc Surg 2017. [Epub ahead of print].

32. Volguina IV, Miller DC, LeMaire SA, et al. Valve-sparing and valve-replacing techniques for aortic root replacement in patients with Marfan syndrome: Analysis of early outcome. J Thorac Cardiovasc Surg 2009;137:1124-32.

33. Zehr KJ, Matloobi A, Connolly HM, et al. Surgical management of the aortic root in patients with Marfan syndrome. J Heart Valve Dis 2005;14:121-8; discussion 8-9.

34. Eagle KA. Rationale and design of the National Registry of Genetically Triggered Thoracic Aortic Aneurysms and Cardiovascular Conditions (GenTAC). Am Heart J 2009;157:319-26.

35. Hughes GC, Zhao Y, Rankin JS, et al. Effects of institutional volumes on operative outcomes for aortic root replacement in North America. J Thorac Cardiovasc Surg 2013;145:166-70.

36. David TE, David CM, Manlhiot C, et al. Outcomes of aortic valve-sparing operations in Marfan Syndrome. J Am Coll Cardiol 2015;66:1445-53.

37. David TE, David CM, Feindel CM, et al. Reimplantation of the aortic valve at 20 years. J Thorac Cardiovasc Surg 2017;153:232-8. 


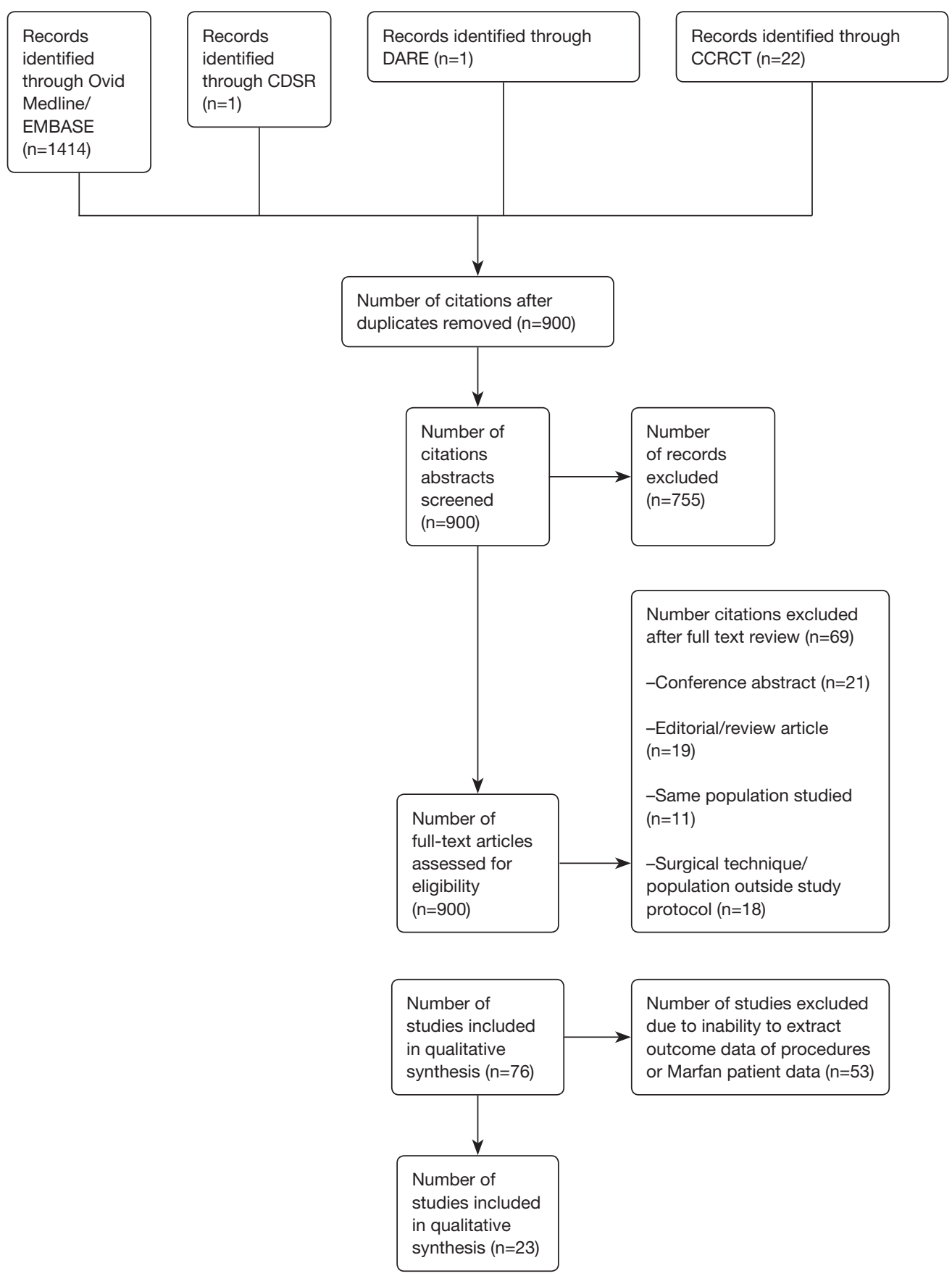

Figure S1 Study selection. Flow chart detailing the steps of systematic review to identify studies reporting the outcomes of composite valve graft and valve sparing root replacement in Marfan syndrome. 


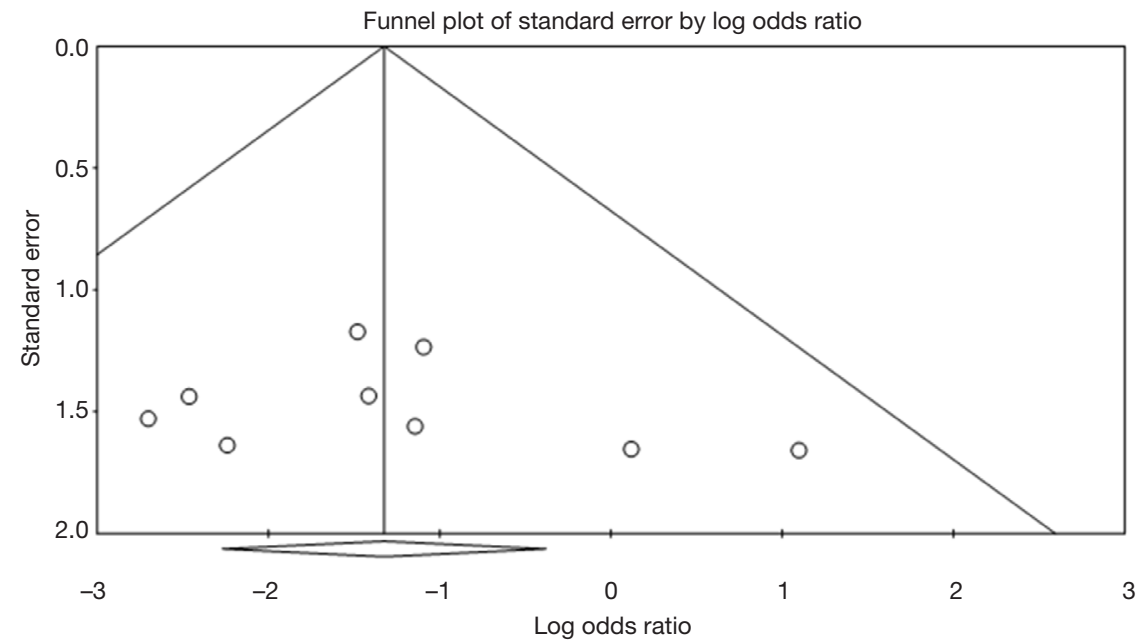

Figure S2 Funnel plot of studies reporting endocarditis rates in VSRR and CVG (Egger's test 1-tail $\mathrm{P}=0.27$ ). VSRR, valve sparing root replacement; CVG, composite valve graft.

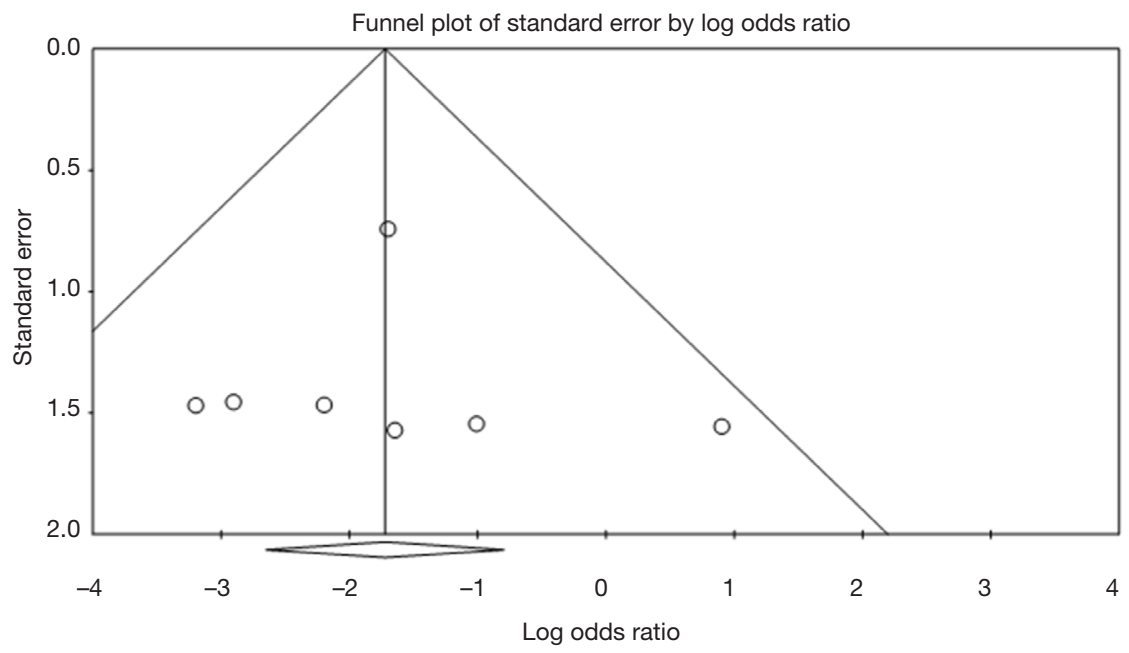

Figure S3 Funnel plot of studies reporting late bleeding events in VSRR and CVG (Egger's test 1-tail P=0.45). VSRR, valve sparing root replacement; CVG, composite valve graft. 


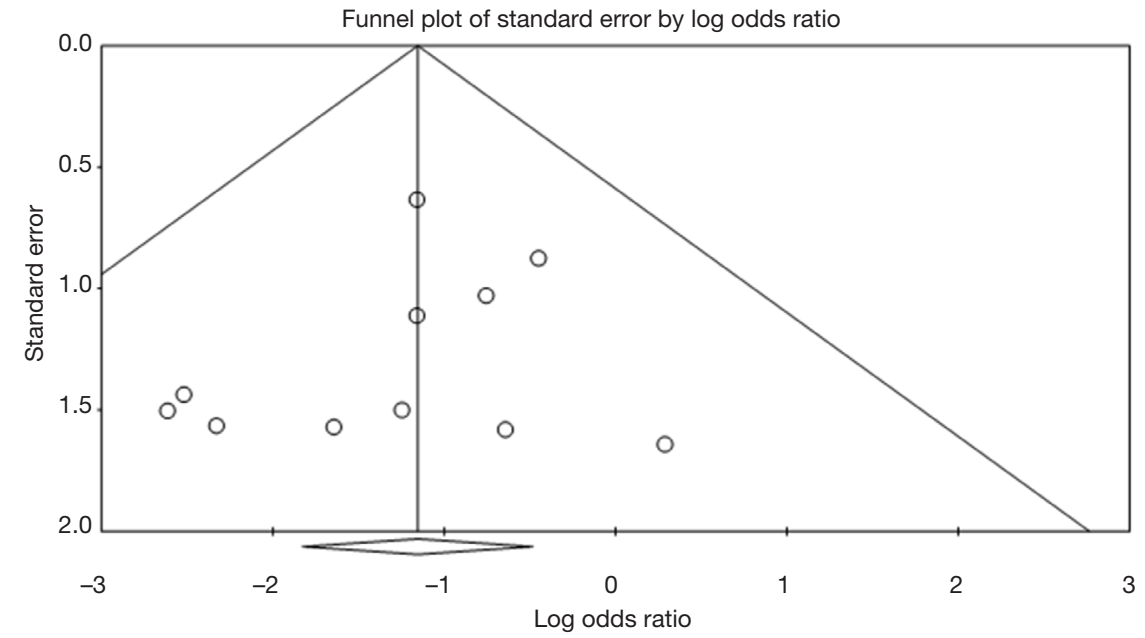

Figure S4 Funnel plot of studies reporting thromboembolic events in VSRR and CVG (Egger's test 1-tail P=0.19). VSRR, valve sparing root replacement; CVG, composite valve graft.

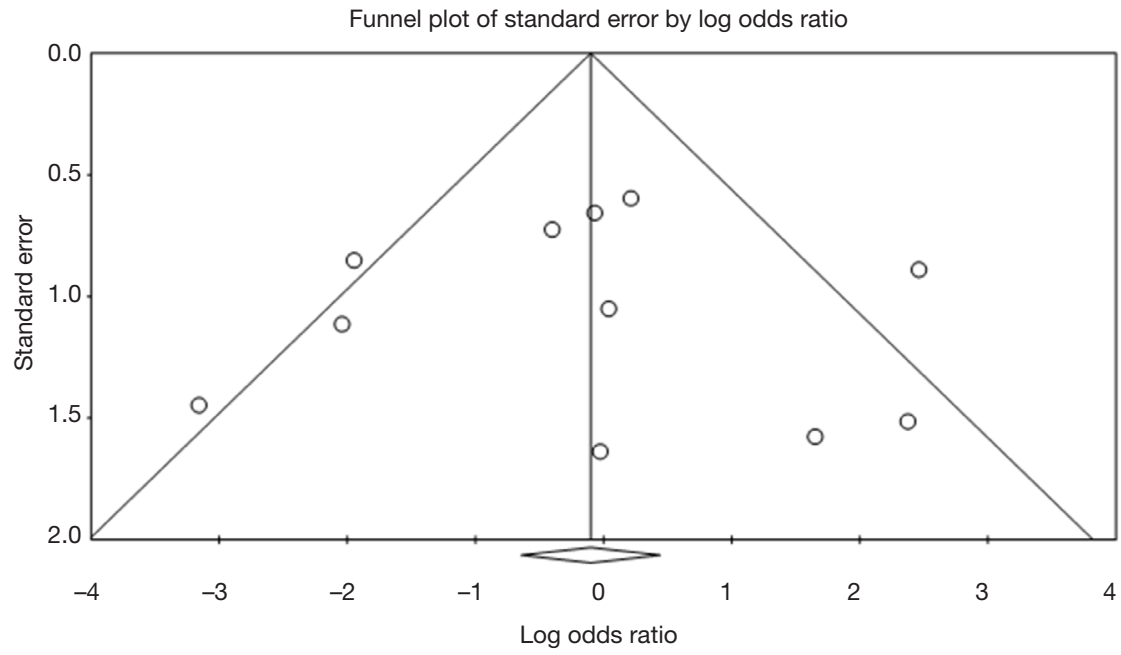

Figure S5 Funnel plot of studies reporting reintervention events in VSRR and CVG (Egger's test 1-tail P=0.49). VSRR, valve sparing root replacement; CVG, composite valve graft. 


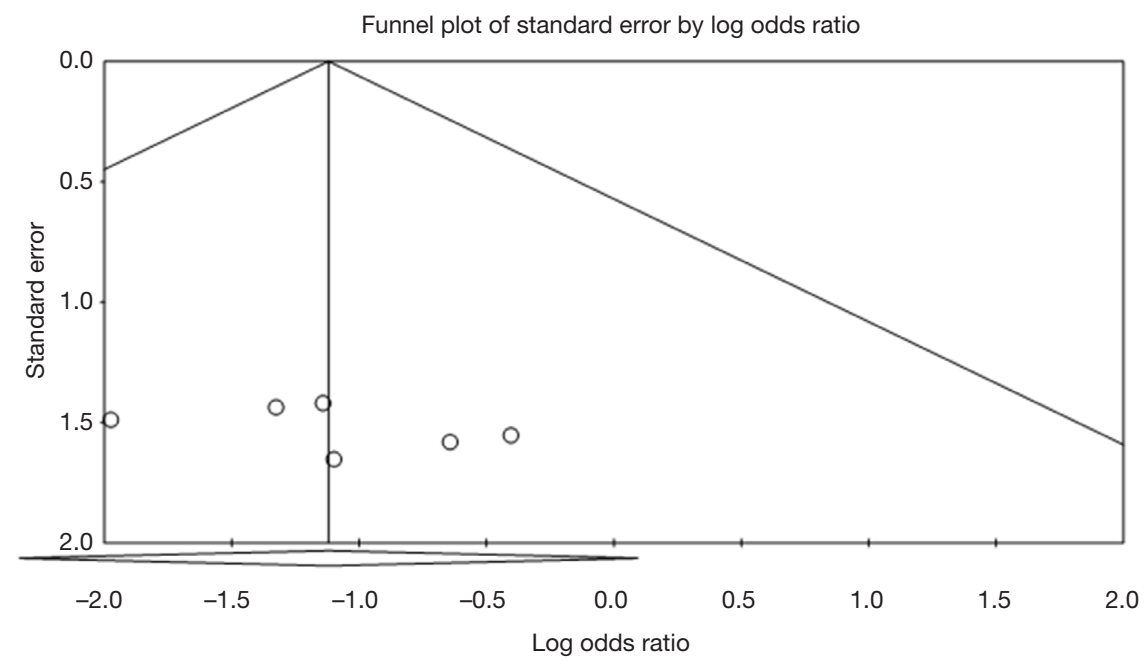

Figure S6 Funnel plot of studies reporting early mortality in VSRR and CVG (Egger's test 1-tail $\mathrm{P}=0.21$ ). VSRR, valve sparing root replacement; CVG, composite valve graft.

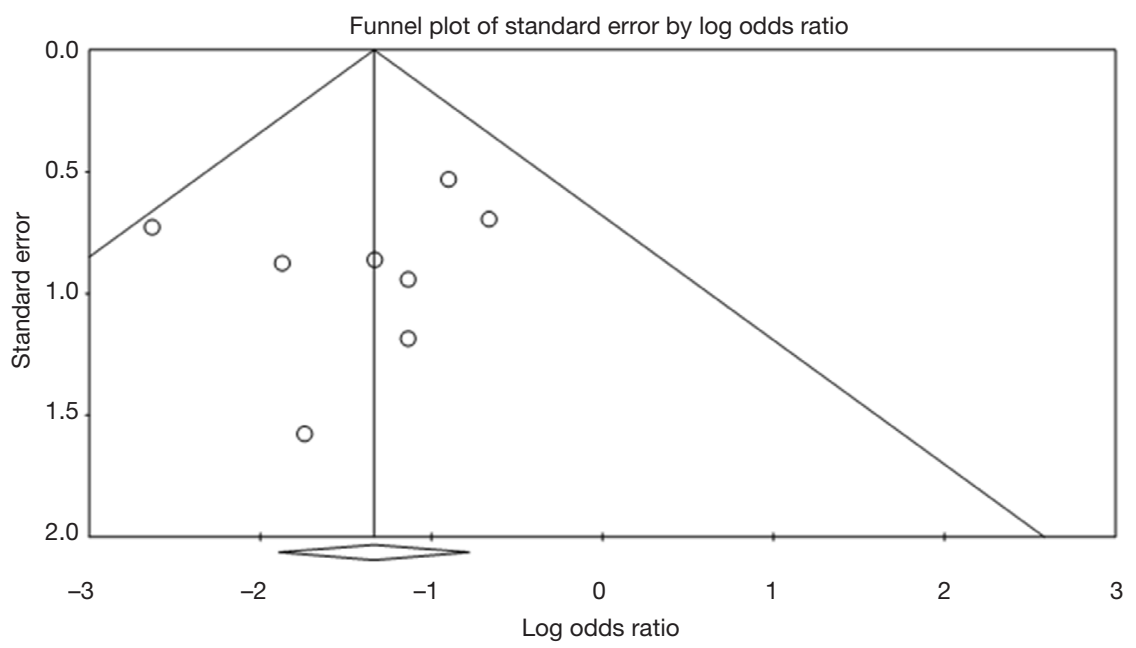

Figure S7 Funnel plot of studies reporting late mortality in VSRR and CVG (Egger's test 1-tail P=0.27). CVG, composite valve graft; VSRR, valve sparing root replacement. 